\title{
Consideraciones de calidad de servicio para tráfico de video en redes wan ${ }^{1}$
}

\section{Quality considerations in the service for video traffic over wan net}

\section{Considerações de qualidade de serviço para o tráfego de vídeo em redes wan}

Recibido: noviembre de 2011

Saira Esperanza Carvajal Ladino ${ }^{2}$

Aceptado: marzo de 2012

Julio Barón Velandia ${ }^{3}$

\section{Resumen}

Este artículo presenta la investigación realizada sobre consideraciones de calidad de servicio para tráfico de video en redes wan,realizado en la Facultad de Ingeniería, en el proyecto curricular de Ingeniería de Sistemas, en él se presentanlas consideraciones para el despliegue de la calidad del servicio en una red en la que se transmite video streaming, incluida la información sobre la clase de servicio, las definiciones de diferentes grupos de tráfico y los procedimientos de buenas prácticas.

Palabras clave: calidad de servicio, clases de servicio, DSCP, probabilidad de pérdida, RFC-4594, TOS, video streaming

\begin{abstract}
This paper is part of the research project that bears his name, held at the Faculty of Engineering, in the Engineering Curriculum Project Systems presents the considerations for deploying quality of service in a network in which transmits streaming video, including information on the service class, the definitions of different groups of traffic, and best practice procedures.
\end{abstract}

Keywords: classes of service, DSCP, loss probability, Quality of Service, RFC-4594, TOS, video streaming,

\section{Resumo}

Este artigo é parte do projeto de pesquisa que leva seu nome, realizado na Faculdade de Engenharia, nos Sistemas de Projecto Curricular de Engenharia apresenta as considerações para a implantação de qualidade de serviço em uma rede na qual transmite streaming de vídeo, incluindo informações sobre a classe de serviço, as definições dos diferentes grupos de tráfego e os procedimentos de melhores práticas.

Palavras Chave- Qualidade de serviço, tipos de serviço, DSCP, probabilidade de perda, RFC-4594, TOS, vídeo Streaming.

1 Artículo de investigación. Resultado del proyecto de investigación realizado en Facultad de Ingeniería de la Universidad Distrital Francisco José de Caldas.

2 Ingeniera de Sistemas. Universidad Distrital Francisco José de Caldas. Bogotá, Colombia. Contacto:sairaecl@yahoo.com

3 Profesor Facultad de Ingeniería. Universidad Distrital Francisco José de Caldas Bogotá, Colombia. Contacto: jbaron@udistrital.edu.co 


\section{Introducción}

El acelerado crecimiento de la Internet y la intranet corporativa, la extensa variedad de nuevas aplicaciones que consumen una gran cantidad de ancho de banda, y la convergencia de datos, voz y tráfico de vídeo sobre la infraestructuras IP, han tenido un gran impacto en la capacidad de las redes para proporcionar servicios garantizados a estas aplicaciones. Para lograr el buen manejo de una infraestructura sobre la que trabajan las aplicaciones de negocio de extremo a extremo es necesario obtener la calidad de servicio requerida a través de una adecuada gestión de los retrasos en la red, requisitos de ancho de banda y parámetros de pérdida de paquetes, mientras que se mantiene la simplicidad y escalabilidad (Cisco System, 2003)

La calidad de servicio QoS (Quality of Service), es un conjunto de estándares en desarrollo que proporcionan procedimientos para dar un trato preferente a determinados tipos de tráfico IP (IBM, 2012).

Ninguna técnica proporciona QoS suficiente y confiable de manera óptima, en su lugar, se han desarrollado una variedad de estrategias con soluciones prácticas que con frecuencia combinan múltiples técnicas (Tanenbaum and Wetherall, 2010).

Para implementar QoS se cuenta con una arquitectura básica que consta de tres elementos fundamentales:

- Técnicas de identificación y marcado para la coordinación de QoS de extremo a extremo entre los elementos de la red.

- QoS en un sólo elemento de red - por ejemplo, colas, y herramientas de modelado de tráfico-.

- Políticas de QoS, gestión, así como funciones de control y administración de extremo a extremo a través de una red de tráfico (Cisco System, 2012).

\section{Precedencia ip y servicios diferenciados (diffserv)}

DiffServ divide el flujo de tráfico en diferentes clases y asigna los recursos de la red con base en las clases definidas en las políticas de QoS. El flujo de tráfico está definido por cinco elementos: dirección IP de origen, dirección IP de destino, puerto de origen, puerto de destino y el protocolo de transporte (Wittenberg, 2009).

La cabecera IP tiene un campo llamado Tipo de Servicio (TOS) que se encuentra entre el campo longitud de la cabecera y el total de longitud de campo. Tradicionalmente, la precedencia IP ha utilizado los tres primeros bits del campo TOS para dar ochovalores posibles de prioridad(DARPA, 1981):

- 000 (0)- Routine (Rutina)

- 001 (1)- Priority (Prioridad)

- 010 (2)- Immediate (Inmediata)

- 011 (3)- Flash

- 100 (4)- Flash Override (Anular Flash)

- 101 (5)- Critical (Crítico)

- 110 (6)- Internetwork Control (Control de Internetwork)

- 111 (7)- Network Control (Control de Red)

DiffServ introduce el concepto de Punto de Código DiffServ (DSCP) que utiliza los seisprimeros bits del campo TOS dando $2^{6}=64$ valores diferentes. EnNichols, Blake, Baker y Black (1998) se describen los servicios diferenciados (DS) y el campo DiffServ Code Point (DSCP).

Con DiffServ, los routers manejan cada paquete de forma diferente dando lugar al concepto de comportamiento de reenvío por salto (PHB), esto es en realidad una forma de transmisión de un flujo particular o grupo de flujos - de agregado de comportamiento-de tráfico en un nodo DiffServ. Un flujo o flujos de paquetes marcados con un DSCP particular en el campo DS se sujeta a un determinado método de envío y reglas que encapsula en el agregado de comportamiento. Este agregado tiene tres elementos, que determinan si la interfaz del router: 1) libera el datagrama, 2) envía el datagrama o 3) se reclasifica, este marcador se detalla enHeinanen, Finland y Guerin (1999). Por ejemplo, cinco flujos pueden ser tratados como un agregado de comportamiento para que sean tratados de manera similar. Cada flujo 
se caracteriza por una probabilidad adicional de descarte y comportamiento de reenvío. La tabla 1 ilustra los valores DSCP. La precedencia IP es definida por los primeros tres bits del campo y la probabilidad de perdida es definida por los siguientes tres bits;por consiguiente, todos los flujos marcados AF1X tienen la misma precedencia de 1 y todos los flujos marcados AFY2 tienen una probabilidad de perdida media.

\begin{tabular}{|c|c|c|c|}
\hline Comportamiento por salto (PHB) & Binario & Precedencia IP & Probabilidad de perdida \\
\hline Default & 000000 & 0 & \\
\hline CS1 & 001000 & \multirow[t]{4}{*}{1} & \\
\hline AF11 & 001010 & & Baja \\
\hline AF12 & 001100 & & Media \\
\hline AF13 & 001110 & & Alta \\
\hline CS2 & 010000 & \multirow[t]{4}{*}{2} & \\
\hline AF21 & 010010 & & Baja \\
\hline AF22 & 010100 & & Media \\
\hline AF23 & 010110 & & Alta \\
\hline CS3 & 011000 & \multirow[t]{4}{*}{3} & \\
\hline AF31 & 011010 & & Baja \\
\hline AF32 & 011100 & & Media \\
\hline AF33 & 011110 & & Alta \\
\hline CS4 & 100000 & \multirow[t]{4}{*}{4} & \\
\hline AF41 & 100010 & & Baja \\
\hline AF42 & 100100 & & Media \\
\hline AF43 & 100110 & & Alta \\
\hline CS5 & 101000 & \multirow[t]{2}{*}{5} & \\
\hline EF & 101110 & & \\
\hline CS6 & 110000 & 6 & \\
\hline CS7 & 111000 & 7 & \\
\hline
\end{tabular}

Tabla 1. Valores DSCP

Fuente: elaboración propia

\section{Requerimientos de QoS para video streaming}

El video streaming es una tecnología basada en el modelo Cliente-Servidor que permite la distribución de multimedia en vivo o grabado previamente. El programa se transmite por la red como un flujo continuo de información que es reproducida por las aplicaciones cliente. Algunas de las aplicaciones son: transmisión de audio y video a través de la Internet o una red privada, entretenimiento, conciertos musicales, eventos deportivos, congresos, video conferencia, telefonía sobre IP, educación, turismo y promociones. Video streaming es usado para broadcasting de canales de video, el cual es comúnmente entregado como IP multicast, y para video bajo demanda (VOD), el cual es entregado como IP unicast.

Si no se emplean técnicas en contra de la pérdida de paquetes, se genera un impedimento visible en el resultado final, pero todo depende de la trama que se pierda en el camino (Rijo Sciara, 2004).

Los valores de la tabla 2 corresponden al informe de ITU-T Y.1541 (International Telecommunication Union, 2011) para requerimientos del video streaming. 


\begin{tabular}{|l|l|l|l|l|}
\hline Aplicación & \multicolumn{4}{|l|}{ Límite superior de parámetros QoS } \\
\hline & Retardo & Jitter & Pérdida & Error \\
\hline $\begin{array}{l}\text { Transacciones } \\
\text { pequeñas, bulk } \\
\text { data, video } \\
\text { streaming, VoD } \\
\text { en disco local }\end{array}$ & $1 \mathrm{~s}$ & Indefinido & $1 \times 10^{-3}$ & $1 \times 10^{-4}$ \\
\hline
\end{tabular}

Tabla 2. Control QOS RED IP[ITU-TY.1541]

Fuente: elaboración propia

\section{Identificación y marcado de QoS}

Para proporcionar servicio preferencial a un tipo de tráficoeste debe:en primer lugar,ser identificado yen segundo lugar, el paquete puede o no estar marcado. Estas dos tareas constituyen la clasificación. Cuando el paquete es identificado, pero no marcado, se dice que la clasificación está en una base por salto (per hop). Es decir, cuando la clasificación se mantiene sólo en el router en el que se realiza, pero no pasa al siguiente router, esto ocurre con Priority Queuing (PQ) y Custom Queuing (CQ). Cuando los paquetes están marcados para uso en toda la red, los bits de precedencia IP pueden ser establecidos.

Entre los métodos más comunes de identificación de los flujos se incluyen: listas de control de acceso (ACL), enrutamiento basado en políticas, tasa de acceso comprometido (CAR), y reconocimiento de aplicaciones basados en la red (NBAR) (Cisco Systems, 2012).

Durante un tiempo, las normas RFC (Request for Comments) del IETF (Internet Engineering Task Force) proporcionaron un conjunto coherente de PHBs para el marcado de aplicaciones, pero no especificaron los valores DSCP para marcar las aplicaciones, generando confusión y desacuerdo sobre los valores que debían asignarse a los diferentes tipos de aplicaciones;para solucionar este problema, en el año 2002 Cisco definió una línea base (Cisco System, 2005). Como se presenta en la tabla 3, al video streaming se le asignó un comportamiento por salto de CS4 (Nichols, Blake, Baker y Black, 1998), esto quiere decir que tiene una precedencia IP de 4 y no tiene asignada probabilidad de perdida.

\begin{tabular}{|c|c|c|c|}
\hline \multirow{2}{*}{ Application } & \multicolumn{2}{|c|}{ L3 Classification } & \multirow{2}{*}{$\begin{array}{c}\text { Referencing } \\
\text { Standard }\end{array}$} \\
\cline { 2 - 3 } & PHB & DSCP & RFC 2474-4.-2.-2 \\
\hline IP Routing & CS6 & 48 & RFC 3246 \\
\hline Voice & EF & 46 & RFC 2597 \\
\hline Interactive-Video & AF41 & 34 & FFC 2474-4.2.2 \\
\hline Streaming Video & CS4 & 32 & RFC 2597 \\
\hline Mission Critical & AF31 & 26 & RFC 2474-4.2.2 \\
\hline Call-Signaling & CS3 & 24 & RFC 2597 \\
\hline Transactional Data & AF21 & 18 & RFC 2474-4.2.2 \\
\hline Network Mgmt & CS2 & 16 & RFC 2597 \\
\hline Bulk Data & AF11 & 10 & Internet 2 \\
\hline Scavenger & CS1 & 8 & RFC 2474-4.1 \\
\hline Best Effort & 0 & 0 &
\end{tabular}

Tabla 3. Línea base QoS DE CISCO Cisco.

Fuente: Cisco System, 2005

Cuatro años después de que Cisco presentó su documento de línea de base de calidad de servicio, el RFC-4594 (Babiarz, Chan y Baker, 2006)fue explícitamente aceptado como un conjunto formal de las mejores prácticas de configuración de DiffServ de QoS. Entre las modificaciones más significativas realizadas en relación con el video están:

- Cambio de los nombres de Interactive Video a Multimedia Conferencing y de Streaming Video a Multimedia Streaming

- La recomendación de Cisco para la marcación Streaming Video, CS4, para marcar Multimedia Streaming con AF31, que se presentan en la tabla 4. Este cambio implica que se le da un tratamiento inferior a los paquetes y que se le asigna una probabilidad de descarte.

- Se adicionó una nueva clase de video "RealTime Interactive”, la cual se marcó CS4. Esto se hizo para diferenciar entre un video de telefonía de menor calidad, referida como Multimedia Conferencing y una aplicación de video de mayor calidad como la telepresencia (Verdantix, 2010). Multimedia Conferencing es marcada como AF41.

- La segunda clase de vídeo adicionado fue "Broadcast Video", marcada como CS3. Esto se hizo para diferenciar entre video streaming de escritorio de menor calidad (Multimedia Streaming) y aplicaciones de difusión de vídeo de mayor calidad como broadcast TV. Multimedia Streaming utiliza la clase AF31 y Broadcast Video utiliza la clase CS3 (Cisco System, 2012). 


\begin{tabular}{|c|c|c|c|}
\hline \multirow{2}{*}{ Application } & \multicolumn{2}{|c|}{ L3 Classification } & IETF \\
\cline { 2 - 4 } & PHB & DSCP & RFC \\
\hline Network Control & CS6 & 48 & RFC 2474 \\
\hline VolP Telefony & EF & 46 & RFC 3246 \\
\hline Call Signaling & CS5 & 40 & RFC2 474 \\
\hline $\begin{array}{c}\text { Multimedia } \\
\text { Conferencing }\end{array}$ & AF41 & 34 & RFC 2597 \\
\hline Real-time interactive & CS4 & 32 & RFC 2474 \\
\hline Multimedia Streaming & AF31 & 26 & RFC 2597 \\
\hline Broadcast Video & CS3 & 24 & RFC 2474 \\
\hline Low-Latency Data & AF21 & 18 & RFC 2597 \\
\hline OAM & CS2 & 16 & RFC 2474 \\
\hline High-Throughput Data & AF11 & 10 & RFC 2597 \\
\hline Best Effort & DF & 0 & RFC 2474 \\
\hline Low-Priority Data & CS1 & 8 & RFC 3662 \\
\hline
\end{tabular}

Tabla 4. Recomendaciones de marcado.

Fuente: Cisco System, 2005

En la práctica, el número de clases en una red empresarial es mucho menor que el número de clases definidas, tanto en la línea base de Cisco como en la del modelo RFC-4594. Por lo general una red empresarial trabajará entre tresy sieteclases de tráfico.

\section{Modelos de provisión de servicio}

A continuación se muestra el mapeo del modelo de oncetipos de aplicaciones de Cisco a modelos de aprovisionamiento de ancho de banda de tres, cuatro y cinco clases.

El problema con el modelo de tres clases radica en que carece de recomendación para abastecer al tráfico de video streaming, como se presenta en la figura 1. Por lo tanto este modelo no es adecuado para una red que transmite tráfico de video streaming.

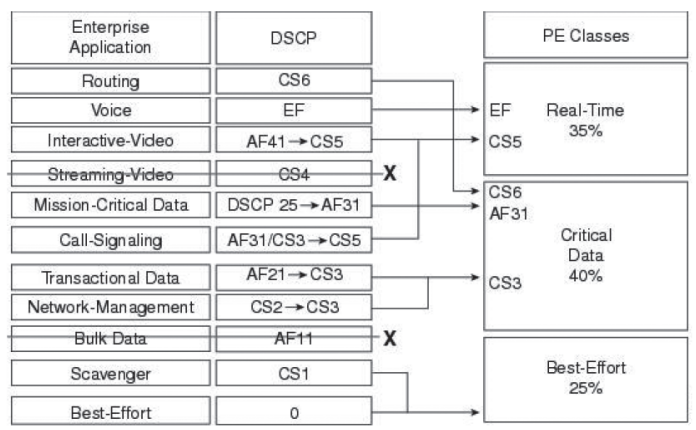

Figura 1. Modelo de provisión de servicios de tres clases.
Con base en el modelo anterior, se construyó un modelo de cuatro clases, como se ve en la figura 2, la clase adicionada abastece el tráfico de video streaming y el tráfico de Network Management, el cual se encontraba en la clase de Critical Data del modelo anterior.

\begin{tabular}{|c|c|c|c|c|}
\hline $\begin{array}{l}\text { Enterprise } \\
\text { Application }\end{array}$ & DSCP & & & PE Classes \\
\hline Routing & CS6 & & \multirow{4}{*}{$\begin{array}{l}\text { EF } \\
\text { CS5 }\end{array}$} & \multirow{4}{*}{$\begin{array}{c}\text { Real-Time } \\
35 \%\end{array}$} \\
\hline Voice & $\mathrm{EF}$ & $\rightarrow$ & & \\
\hline Interactive-Video & $\mathrm{AF} 41 \rightarrow \mathrm{CS} 5$ & \multirow[b]{3}{*}{$\longrightarrow$} & & \\
\hline Streaming-Video & $\mathrm{CS} 4 \rightarrow \mathrm{AF}_{21}$ & & & \\
\hline Mission-Critical Data & $\mathrm{DSCP} 25 \rightarrow \mathrm{AF} 31$ & & \multirow{2}{*}{\begin{tabular}{|l|} 
CS6 \\
AF31 \\
CS3
\end{tabular}} & \multirow{2}{*}{$\begin{array}{l}\text { Critical } \\
\text { Data } \\
25 \%\end{array}$} \\
\hline Call-Signaling & $\mathrm{AF} 31 / \mathrm{CS} 3 \rightarrow \mathrm{CS} 5$ & & & \\
\hline Transactional Data & $\mathrm{AF} 21 \rightarrow \mathrm{CS} 3$ & & \multirow{2}{*}{$\begin{array}{l}\text { AF21 } \\
\text { CS2 }\end{array}$} & \multirow{2}{*}{$\begin{array}{l}\text { Video } \\
15 \%\end{array}$} \\
\hline Network-Man agement & $\mathrm{CS} 2$ & $\longrightarrow$ & & \\
\hline Bulk Data & AF 11 & & \multirow{3}{*}{\multicolumn{2}{|c|}{$\begin{array}{c}\text { Best-Effort } \\
25 \%\end{array}$}} \\
\hline Scavenger & CS1 & & & \\
\hline Best-Effort & 0 & & & \\
\hline
\end{tabular}

Figura 2. Modelo de provisión de servicios de cuatroclases.

Fuente: Cisco System, 2012

Con el modelo anterior es suficiente para realizar la clasificación del tráfico de red, pero si la red transmite otro tipo tráfico diferente a video streaming se requiere un mayor nivel de detalle, la figura $3 \mathrm{se}$ presenta un modelo de cincoclases, dando aprovisionamiento a la clase Bulk Data, lo cual no se había realizado con los modelos anteriores; en esta clase, se encuentran el flujo de aplicaciones FTP y de e-mail (Giusto, Lera, Morabito y Atzori, 2010).

\begin{tabular}{|c|c|c|c|c|}
\hline $\begin{array}{l}\text { Enterprise } \\
\text { Application }\end{array}$ & DSCP & & & PE Classes \\
\hline Routing & CS6 & & \multirow{4}{*}{$\begin{array}{l}\text { EF } \\
\text { CS5 }\end{array}$} & \multirow{4}{*}{$\begin{array}{c}\text { Real-Time } \\
35 \%\end{array}$} \\
\hline Voice & $\overline{E F}$ & 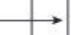 & & \\
\hline Interactive-Video & $\mathrm{AF} 41 \rightarrow \mathrm{CS} 5$ & & & \\
\hline Streaming-Video & $\mathrm{CS} 4 \rightarrow \mathrm{AF} 21$ & \multirow{2}{*}{$\stackrel{\longrightarrow}{\longrightarrow}$} & & \\
\hline Mission-Critical Data & $\mathrm{DSCP} 25 \rightarrow$ AF 31 & & \multirow{2}{*}{$\begin{array}{l}\text { CS6 } \\
\text { AF31 } \\
\text { CS3 }\end{array}$} & \multirow{2}{*}{$\begin{array}{c}\text { Critical } \\
\text { Data } \\
20 \%\end{array}$} \\
\hline Call-Signaling & $\mathrm{AF} 31 / \mathrm{CS} 3 \rightarrow \mathrm{CS} 5$ & \multirow[b]{2}{*}{$\longrightarrow$} & & \\
\hline Transactional Data & $\mathrm{AF}_{21} \rightarrow \mathrm{CS}_{3}$ & & AF21 & Video \\
\hline Network-Management & $\mathrm{CS} 2$ & \multirow{2}{*}{\multicolumn{3}{|c|}{ AF11/CS1 Bulk Data 5\% }} \\
\hline Bulk Data & AF11 & & & \\
\hline Scavenger & $\mathrm{CS} 1 \rightarrow 0$ & & \multirow{2}{*}{\multicolumn{2}{|c|}{$\begin{array}{c}\text { Best-Effort } \\
25 \%\end{array}$}} \\
\hline Best-Effort & 0 & & & \\
\hline
\end{tabular}

Figura 3. Modelo de provisión de servicios de cincoclases.

Fuente: Cisco System, 2012 


\section{Discusión}

Con el paso del tiempo, la clasificación para el marcado y el aprovisionamiento de ancho de banda del tráfico de streaming de video ha variado, permitiendo mejorar la calidad de servicio de los diferentes flujos transmitidos por la red. Dado que la calidad de servicio está altamente relacionadacon el tipo de red donde debe ser aplicado, el estudio de los actuales modelos y recomendaciones es fundamental para proponer mejoras y generación de nuevos modelos que proporcionen condiciones exigentes de calidad a los diferentes flujos requeridos por los nuevos tipos de aplicaciones que demandan los diferentes tipos de clientes.

El manejo y caracterización de los flujos de tráfico permitirá el desarrollo de aplicaciones que faciliten la negociación dinámica de recursos en las diferentes topologías involucradas en el consumo de video streaming.

\section{Referencias}

Babiarz, J.; Chan, K. and Baker, F. (2006). Guidelines for DiffServ Service Classes. The Internet Society.

Cisco System, Inc. (2003). Service Provider Qualityof-Service Overview.

Cisco System, Inc. (2005). The QoS Baseline. Available: http://www.cisco.com/en/US/technologies/tk543/tk759/technologies_white_ paper0900aecd80295a9b.pdf

Cisco System, Inc. (2012). Enterprise Medianet Quality of Service Design 4.0-Overview. Available: http://www.cisco.com/en/US/docs/ solutions/Enterprise/WAN_and_MAN/QoS_ SRND_40/QoSIntro_40.html
Cisco Systems, Inc. (2012). Internetworking Technology Handbook.

DARPA. (1981). IETF TOOLS.Acces: septembre. Available: http://tools.ietf.org/html/rfc791

Giusto, D.; Lera, A.; Morabito, G. and Atzori, L. (2010). The Internet of Things: 20th Tyrrhenian Workshop on Digital Communications. New York: Springer-Verlag.

Heinanen, J.; Finland, T. and Guerin, R. (1999). A Single Rate Three Color Marker.Acces: septembre.Available: http://tools.ietf.org/html/rfc2697

IBM. (2012). Modelos QoS. Acces: 23 novembre, 2012. Available: http://publib.boulder. $\mathrm{ibm} . c o m /$ infocenter/pseries/v5r3/index. jsp?topic $=/$ com.ibm.aix.commadmn $/ \mathrm{doc} /$ commadmndita/sctp_tcpip_qos_models.htm

International Telecommunication Union. (2011). Network performance objectives for IP-based services. ITU-T.

Nichols, K.; Blake, S.; Baker, F. and Black, D. (1998). Definition of the Differentiated Services Field (DS Field). Acces: decembre. Available: http:// tools.ietf.org/html/rfc2474

Rijo Sciara, D. (2004). Fundamentos de Video Streaming. Montevideo: Uruguay.

Tanenbaum, A. S. and Wetherall, D. S. (2010). Computer Networks. Boston: Prentice Hall.

Verdantix. (2010). The Telepresence Revolution. Carbon Disclosure Project.

Wittenberg, N. (2009). Understanding Voice Over IP Technology. New York: Cengage Learning. 